Infektionen

\section{Unterscheidung von infektiöser und nicht- infektiöser Arthritis des Handgelenks}

Kang $\mathrm{G}$ et al. Wrist inflammation: a retrospective comparison between septic and non-septic arthritis. J Hand Surg Eur Vol 2018; 43: 431 - 437

Eine Entzündung des Handgelenks ist ein relativ häufiger Befund und kann vielfältige Ursachen haben. Wichtig ist das schnelle Erkennen einer infektiösen Genese, um weitreichende Folgen wie dauerhafte Gelenkschäden und im Extremfall sogar eine generalisierte Sepsis zu verhindern.

Umgekehrt würde eine große Zahl von Patienten einem unnötigen operativen Eingriff unterzogen, wenn zur Diagnosesicherung routinemäßig eine chirurgische Eröffnung des Gelenks erfolgen würde.

Die klinische Differenzialdiagnose kann aber schwierig sein: Weder die körperliche Untersuchung noch schnell verfügbare Laborparameter können eine infektiöse Genese eindeutig sichern oder ausschließen. Und bis zum Vorliegen mikrobiologischer Ergebnisse vergeht wertvolle Zeit. Eine Gruppe aus Singapur hat nun Patienten mit später gesicherter bzw. später ausgeschlossener Infektion des Handgelenks einander gegenübergestellt und nach Unterschieden zwischen diesen beiden Gruppen gesucht.

Die Mediziner um Gavrielle Kang haben dazu zwischen Mai 2012 und April 2015 Daten von 77 stationär in ihrer Klinik behandelten Patienten retrospektiv ausgewertet. Darunter waren laut Abschlussdiagnose

- 15 Patienten mit gesicherter Infektion des Handgelenks (20\%; Gruppe 1), im Wesentlichen durch grampositive Erreger, und

- 62 Patienten mit gesicherter nichtinfektiöser Arthritis (80\%; Gruppe 2), v.a. aufgrund akuter Exazerbation einer Gicht oder Pseudogicht ( $n=57)$.
Patienten mit akuten Frakturen und Traumata waren von der Auswertung ausgeschlossen. Patienten der Gruppe 2 wiesen signifikant häufiger

- eine chronische Niereninsuffizienz (37,1\% vs. $0 \%$ in Gruppe 1 ),

- eine anamnestisch bekannte Gichterkrankung (54,8\% vs. $13,3 \%$ ) und

- erhöhte Harnsäurekonzentrationen im Serum (37,1\% vs. $0 \%)$. auf.

Umgekehrt fanden sich bei Patienten der Gruppe 1 signifikant häufiger

- erhöhte Werte bei $\geq 2$ von 3 Entzündungsparametern (C-reaktives Protein [CRP], Leukozytenzahl, Erythrozytensedimentationsgeschwindigkeit [ESG]) bei gleichzeitig normaler Harnsäurekonzentration ( $100 \%$ vs. $50 \%$ in Gruppe 2).

Dagegen zeigten sich zwischen den Gruppen keine Unterschiede bei der Häufigkeit eines Diabetes mellitus und der Häufigkeit immunsuppressiver Erkrankungen und/oder Behandlungen (z. B. aktive Malignom unter Zytostatikatherapie und rheumatologische Erkrankungen unter Steroidtherapie). Ebenso schloss die Beteiligung mehrerer Gelenke eine infektiöse Ursache nicht aus, dagegen war der Nachweis einer Chondrokalzinose im Standard-Röntgenbild des Handgelenks nahezu beweisend für eine nicht-infektiöse Arthritis.

In einer Subgruppenanalyse, in die nur Patienten mit ausschließlicher Handgelenkentzündung ohne Begleiterkrankungen eingeschlossen wurden, fanden die Forscher darüber hinaus einen signifikanten Unterschied bei den CRP-Konzentrationen: Der Mittelwert betrug 218 $\mathrm{mg} / \mathrm{l}$ in Gruppe 1 gegenüber 85,6 mg/l in Gruppe 2.
FAZIT

Die schnelle Abgrenzung von infektiösen gegenüber nicht-infektiösen Ursachen einer Handgelenkentzündung bleibt nach diesen $\mathrm{Er}$ gebnissen weiterhin schwierig, fassen die Autoren zusammen. Manche Befundkombinationen sprechen allerdings eher für die eine oder die andere Genese. Ihre Untersuchung könnte Anhaltspunkte für die Planung einer zukünftigen prospektiven Studie liefern, so Kang et al. abschließend, die Klinikern mehr Sicherheit bei der Differenzialdiagnose verleiht.

Dr. Elke Ruchalla, Bad Dürrheim 